\title{
Por un enfoque psicolingüístico del aprendizaje de la lectura y sus dificultades ${ }^{1}$
}

\section{Jesús Alegría*}

Universidad Libre de Bruselas

\section{COMPRENSION DE LA LENGUA ORAL Y DE LA LENGUA ESCRITA.}

Leer, lo mismo que comprender un mensaje oral, es un acto que hace intervenir una serie de competencias de naturaleza lingüistica. Al comenzar el aprendizaje de la lectura, alrededor de los seis años, el niño posee la competencia lingüistica necesaria para producir frases perfectamente inteligibles y comprender las que llegan a sus oídos. Aprender a leer va a consistir para él en descubrir cómo utilizar parte de este dispositivo destinado inicialmente a ocuparse de la lengua oral a partir de informaciones visuales, es decir, del texto escrito. El resultado final. del proceso de lectura, la comprensión del mensaje escrito, es idéntico: al obtenido cuando el mensaje llega por la vía oral. Las diferencias que: existen entre las dos formas de alcanzar el significado provienen de los aspectos específicos de cada una de ellas.

Una razón importante para interrogarse sobre la especificidad de la lectura respecto a la lengua oral es pedagógica. Aprender a hablar y comprender la palabra no plantea problemas salvo en algunos casos excepcionales de carácter patológico grave. Todos los niños, sea cual sea su lengua materna, medio socio-económico, nivel intelectual, etc., aprenden a hablar en la medida en que ciertas condiciones mínimas de exposición son respetadas. La adquisición del lenguaje se hace de manera similar en todos los casos y no necesita la intervención de instituciones socialmente organizadas. $\mathrm{La}$ adquisición de la lectura es diferente. En primer lugar no se produce espontáneamente. El hecho de estar rodeado de mensajes escritos no conduce al niño, ni tampoco al adulto, a aprender a leer. La consecuencia es que la sociedad ha institucionalizado el aprendizaje. En segundo lugar, los resultados no son siempre satisfactorios. Un número importante de niños no consi-

* Directión del autor: Unniversité Libre de Bruxelles. Laboratoire de Psychologie Expérimentale, i 17 Av. Ad. Buyl, roso Bruxelles, Bélgica. 
gue aprender a leer de manera adecuada. Esta dificultad no puede ser en muchos casos atribuida a dificultades generales de aprendizaje. La noción de dislexia corresponde exactamente a esta realidad. Esto nos lleva naturalmente a preguntarnos: ¿qué tiene el mensaje escrito que lo hace tanto más difícil que el mensaje oral para un número importante de individuos? Si pudiéramos formular una respuesta clara a esta pregunta habríamos dado un paso adelante considerable en la comprensión de la naturaleza de las dificultades de muchos niños, y por consiguiente en la concepción de formas eficaces para corregirlas. En este artículo vamos a examinar dos maneras de considerar el problema de la especificidad de la lectura respecto a la lengua oral. Una de ellas es de orientación perceptiva y la otra lingüistica. No tengo la pretensión de poder dar una respuesta clara y definitiva a la pregunta que acabo de formular, el problema es demasiado complejo. Me limitaré aquí a examinar algunos de los trabajos realizados en los últimos diez años por la psicología cognitiva y la psicolingüística que permiten responder de manera categórica a ciertos aspectos de nuestra pregunta.

\section{DIFICULTADES PERCEPTIVAS EN LOS MALOS LECTORES.}

Una fuerte tradición en psicologia de la lectura supone que las dificultades de aprendizaje son de origen perceptivo-visual. El razonamiento de esta corriente es el siguiente: si un niño tiene dificultades para aprender a leer es porque sus mecanismos de tratamiento de la información visual en general son deficientes. El texto se presenta al lector como una serie de signos gráficos u ortográficos, que dan acceso al mensaje lingüístico a condición de ser analizados correctamente. Una deficiencia a este nivel va a crear irremediablemente dificultades de comprensión. La confusión entre las letras $b, d, p$ y $q$ por ejemplo, según esta teoría, es de origen perceptivo-visual o visuo-espacial. La interpretación de errores de lectura que consisten en intervertir letras, leer los en vez de sol por ejemplo, es idéntica. El lector tiene dificultades con la organización espacial (izquierda-derecha) del tratamiento de la información visual. Estos problemas van a menudo acompañados de dificultades con el llamado esquema corporal. A la base de esta concepción de las dificultades de lectura aparece la idea (no siempre explicita) de que su origen causal es neurológico. Ciertos autores son más precisos aún y proponen modelos de funcionamiento cerebral más - menos aproximados, en los cuales el tema dominante es una insuficiencia y/o un atraso de la lateralización hemisférica ${ }^{2}$.

Esta teoria tiene una influencia considerable a nivel de la práctica pedagógica y terapéutica. Exagerando apenas, si un niño confunde la $b$ y la $d$ a los siete años se arriesga a ser considerado disléxico y a cargar un diagnóstico de problemas neurológicos probables, calificados púdicamente de mínimos, en la medida en que no son detectables. El tipo de terapia de la lectura al que esta teoría conduce consiste en hacer practicar al niño ejercicios destinados a mejorar su percepción visual y su coordinación visomotora, sobre todo en relación con la organización espacial en términos de izquierda-derecha. El sentido común indica que todo tiene una relación muy lejana con la lectura (si tiene alguna en 
que personas que poseen una visión y una coordinación visomotora

excepcionalmente buenos, como ciertos deportistas, por ejemplo, son pésimos lectores, mientras que sus hermanos o hermanas totalmente incapaces de lanzar una pelota con cierta precisión, son lectores ávidos y de gran habilidad. Los trabajos de Vellutino y sus colaboradores en la Universidad de Chicago demuestran más allá de toda duda razonable que los niños con problemas de aprendizaje de la lectura no tienen problemas de percepción visual ni visuo-espacial. Esta conclusión se opone a ideas profundamente ancladas en la mente de muchos psicólogos y pedagogos y conviene detenerse un momento para examinar con cierto detalle las experiencias en las cuales está basada.

El primer problema que encontramos para abordar el estudio de las causas eventuales de la dislexia es la definición del grupo. Esta definición es, desgraciadamente, negativa. Es disléxico un niño que no aprende a leer normalmente siendo que en apariencia no le falta nada por adquirir esta habilidad: $n i$ inteligencia, por ejemplo en aritmética progresa normalmente; $n i$ métodos de enseñanza adecuados, sus compañeros de clase progresan normalmente; $n i$ un medio socio-económico apropiado, sus hermanos por ejemplo aprenden o aprendieron normalmente, etc. La idea de base de la definición es que si la dislexia, es decir, una dificultad específica del aprendizaje de la lectura, existe realmente, podremos aprender algo sobre ella estudiando un grupo de niños que no aprende a leer sin que sus dificultades puedan ser atribuidas a factores no específicos. Esto no quiere decir que un niño. poco inteligente o que ha tenido una escolaridad irregular a causa de enfermedades frecuentes, por ejemplo, no pueda ser disléxico. Simplemente no será incluido en el grupo porque sus problemas de lectura podrian ser explicados sin hacer intervenir la dislexia. Vellutino hace pasar una batería de tests de lectura a los niños que reúnen estas condiciones y los incluye en su grupo si presentan un atraso superior o igual a dos años. Con los niños de segundo año, un atraso de un año basta para ser incluidos en el grupo.

El paradigma utilizado por Vellutino para estudiar los eventuales problemas visuo-espaciales potencialmente en la base de las dificultades de lectura, consiste en comparar un grupo de lectores normales con otro. formado de malos lectores. En una condición los sujetos tienen que leer (o nombrar) una serie de letras, cifras o formas geométricas diversas y en la otra condición su tarea consiste en copiarlas. Si los problemas de los malos lectores fueran de origen visual tendriamos que observar una deficiencia de este grupo respecto al grupo de buenos lectores tanto en la condición copia como en la condición lectura, puesto que la percepción visual interviene de manera idéntica en los dos casos. Los resultados pueden ser enunciados de manera simple. Los malos lectores son peores que los buenos cuando se trata de leer letras, cifras y otras configuraciones visuales. Esto no tiene nada de sorprendente puesto que se trata de malos lectores. En la condición copia, sin embargo, los malos lectores no difieren significativamente de los buenos.

El primer trabajo importante en este dominio es de 1972 (Vellutino, Steger y Kandel). Los niños que participaron en la experiencia tenían nueve y catorce años. Los estimulos visuales eran series de tres, cuatro o cinco letras (diny, jnyc, etc.), palabras (was, fróm, etc.), o cifras ( 382 , 4328 , etc.), o bien, series de dos o tres formas geométricas ( $\square \square$, $\mathrm{O} \Delta \mathrm{u}$, etc.). Los estímulos visuales aparecían durante $600 \mathrm{~ms}$ : en una 
pantalla y el niño tenía que leerlos o copiarlos según la condición, en cuanto desaparecían de su vista. A esta experiencia hay que agregar las de Vellutino, Pruzek, Steger y Meshoulam (1973) y Vellutino, Steger, Kaman y DeSetto (1975) construidas con la misma fórmula. Los sujetos tenían siete y doce años. Los estímulos eran palabras de tres, cuatro o cinco letras escritas en hebreo. La presentación duraba tres, cuatro o cinco sec, respectivamente. Los sujetos no conocian el hebreo y por consiguiente las letras eran para ellos configuraciones visuales. En ninguna de estas experiencias los malos lectores difieren de los buenos cuando se trata de copiar configuraciones visuales. Este resultado está en franca contradicción con la idea de que los primeros sean malos lectores porque su sistema de tratamiento de información visual es deficiente.

Más recientemente, Ellis (198I) ha abordado este problema comparando buenos y malos lectores en la situación experimental de. Posner (1969). Los sujetos ven en la pantalla de un taquistoscopio dos letras (o dos formas geométricas en algunas condiciones). Su tarea consistía en responder lo más rápido posible si las dos letras (formas) son iguales o no. Iguales puede ser definido de dos maneras diferentes según la condición: o bien las letras son físicamente iguales, por ejemplo, a-a, o bien tienen el mismo nombre, a-A, aunque sean fisicamente diferentes. Los sujetos examinados por Ellis eran buenos o.malos lectores cuya edad de lectura era de 12,7 y 9,0 años, respectivamente. La edad cronológica de los dos grupos era de 11,8 años. Los resultados muestran que el tiempo de reacción medio para dar la respuesta uiguales» es similar en los dos grupos de sujetos cuando la igualdad está definida como igualdad física $(a=a): 1.05$ I y $1.022 \mathrm{~ms}$ en los malos y buenos lectores, respectivamente. Cuando la igualdad se define como igualdad de nombre $(\mathrm{a}=\mathrm{A})$ el incremento de tiempo de reacción respecto a la igualdad fisica es significativamente superior en los malos lectores que en los buenos: 143 y $49 \mathrm{~ms}$ de incremento, respectivamente. Este resultado demuestra que los malos lectores no tienen problemas para comparar dos configuraciones visuales. Si el origen de sus dificultades de lectura se situara a este nivel hubiéramos observado una diferencia de tiempo de reacción (o de la cantidad de errores) en la condición igualdad fisica entre buenos y malos lectores. La diferencia entre los dos grupos aparece en la condición igualdad de nombre. Esto nos da una pista importante para comprender el origen (o más bien uno de los orígenes) de las dificultades de los malos lectores. La deficiencia de este grupo aparece cuando se trata de nombrar una configuración visual: letra o forma geométrica. En la situación de Ellis, para responder (igual» a los estímulos a-A, por ejemplo, el sujeto tiene que: I) hacer un análisis visual para identificar las configuraciones, 2) encontrar el nombre de cada una de ellas y 3) comparar los dos nombres. Los malos lectores no tienen problemas en la primera operación sino en la segunda o/y en la tercera. Una interpretación idéntica puede darse de los resultados de Vellutino. La diferencia entre buenos y malos lectores aparece únicamente cuando el niño tiene que nombrar un estímulo, es decir, cuando la segunda operación interviene. Estos resultados conducen inevitablemente a la conclusión de que los malos lectores no tienen problemas de percepción visual o de organización de la información visuo-espacial sino en operaciones que hacen intervenir la atribución de un nombre a una configuración visual. 
Si se admite la idea de que las dificultades de los malos lectores no son visuo-espaciales, sino de atribución de un nombre a una configuración visual, ¿cómo entender el origen de esta deficiencia y sus repercusiones en la lectura? Para identificar un objeto, tanto real como representado (foto, dibujo, etc.), del mismo modo que para identificar el nombre escrito, la representación visual (objeto, foto, palabra escrita, etc.), tiene que alcanzar el léxico interno del sujeto. Este léxico es una estructura hipotética donde el individuo tiene almacenados de manera organizada sus conocimientos lexicales, semánticos y sintácticos. El niño de tres años, por ejemplo, que reconoce un pato dibujado, ejecuta una operación de acceso al léxico. El nombre pato que esta operación puede evocar de manera explícita o no, es uno de los resultados de la operación. Otro es la evocación de toda una serie de asociaciones relativas a pato. Un fenómeno semejante tiene lugar cuando la entidad lexical pato es alcanzada por la vía auditiva, es decir, cuando el niño oye esta palabra.

En el caso de la lectura de una palabra el acceso al léxico es más complejo. Aprender a leer consiste en crear un medio que permita acceder al léxico interno a partir/de la representación ortográfica. Esta representación es de tipo alfabético en nuestra cultura. La figura I

\section{FIGURA I}

Representación esquemática de un modelo de identificación de palabras. El modelo supone la existencia: de una entidad lexical a la cual se puede llegar directamente por la vía icónica o fonológica, o indirectamente gracias a un fenómeno de asociación (si oigo laguna pienso en pato $y$ viceversa).

Sea cual fuere el modo de acceso a la entidad lexical, una serie de conocimientos (semánticos, sintácticos, pragmáticos, etc...) son evocados, del mismo modo que otras entidades lexicales asociadas (Donald, laguna, etc.), y el código fonológico correspondiente.

Bajo la linea de puntos aparecen las dos vias de acceso al léxico posibies a partir de la representación ortográfica, la via directa y la via fonologica.

\section{CONOCIMIENTOS}

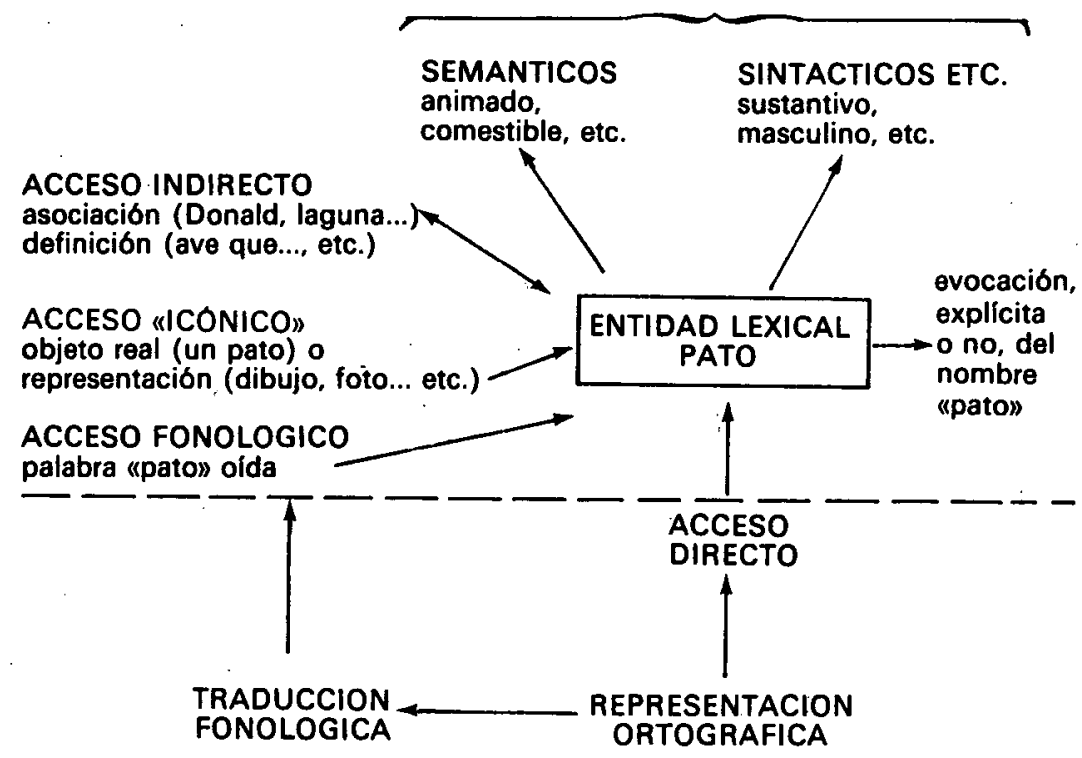


mena representa esquemáticamente el modelo propuesto aquí. La línea de puntos separa los aspectos «naturales» de los aspectos «culturales» de modelo. Siguiendo las ideas expuestas en la introducción, los aspectos naturales hacen referencia a la lengua oral, adquirida espontáneamente por el niño, y sin dificultades. Los aspectos culturales hacen referencia a la lengua escrita, que puede plantear problemas de adquisición. El modelo supone que el punto de encuentro de la lectura y la lengua oral es doble. Una vía de acceso de la representación ortográfica al léxico es directa. La palabra escrita es reconocida como una totalidad no analizada, de la misma forma que se reconoce un número o un signo de interrogación. Lo esencial de esta vía es que no hace intervenir la palabra en las operaciones de acceso al léxico. La otra vía consiste en crear una representación fonológica a partir de la representación ortográfica y usar esta representación para acceder al léxico. En este procedimiento el lector entra en contacto con la vía oral antes de alcanzar el léxico.

Este modelo, presentado de manera simplificada aquí, está apoyado por un gran número de trabajos experimentales y clínicos (ver, por ejemplo, Coltheart, 1978; Baron y Treiman, 1980; Jorm y Share, 1983). Su interés proviene del hecho de que facilita la visualización de los aspectos de la adquisición de la lectura potencialmente deficitarios en el mal lector, y permite dejar de lado otros que son claramente no pertinentes. Por ejemplo, los procesos pos-lexicales que intervienen en la comprensión de frases son los mismos, sea cual sea la forma en que las entidades lexicales que constituyen la frase fueron alcanzadas, por vía oral u ortográfica. Las dificultades de lectura no pueden situarse a ese nivel en la medida en que los procesos pos-lexicales son comunes a la lectura y a la comprensión de la palabra y que los malos lectores comprenden normalmente el mensaje cuando les es presentado oralmente.

El modelo propuesto conduce a preguntarse cómo el niño que aprende a leer va a poder desarrollar las vias que le permiten acceder a sus conocimientos lingüísticos a partir de la representación ortográfica. El problema se plantea diferentemente según el sistema de representación ortográfica considerado. Las culturas que han desarrollado una escritura han dado respuestas diferentes al problema de la representación directa de objetos o conceptos (sistemas logográficos) y a la representación de segmentos de la lengua oral (sistemas fonográficos). El chino moderno es un ejemplo contemporáneo de escritura logográfica: El logograma correspondiente a pato, por ejemplo, no puede ser descompuesto en elementos constituyentes. Lo mismo ocurre con la cifra árabe 3. Tanto en un caso como en el otro sólo existe la vía directa al léxico interno. Este sistema tiene la ventaja de poder ser practicado por personas que hablan lenguas diferentes. $\mathrm{El} /$ es comprendido del mismo modo por un inglès que por un sueco. En China esta ventaja es considerable porque se hablan más de 1.800 dialectos diferentes. $\mathrm{El}$ inconveniente de un sistema logográfico es que para poder escribir todo lo que se puede decir hay que crear un número gigantesco de logogramas. En chino contemporáneo este número es superior a 50.000. Incluso un letrado tiene que recurrir constantemente al diccionario para leer un texto de cierta complejidad.

La introducción de una vía indirecta de acceso al léxico aparece con los sistemas fonográficos. En este caso cada signo ortográfico repre- 
senta un segmento de palabra. Los segmentos pueden ser de tamaño diferente según los sistemas. El japonés actual, por ejemplo, utiliza dos silabarios, el Hiragana y el Katakana, en los cuales cada signo corresponde a una sílaba ${ }^{3}$. La lengua japonesa se presta a este tipo de representación ortográfica porque tiene una estructura fonética formada de secuencias de sílabas consonante-vocal. Cuarenta y seis signos de base permiten escribir todo lo que se puede decir (véase Sakomoto, 1980, para una presentación más detallada). El proceso de segmentación de la palabra no se detiene al nivel de la sílaba. Las sílabas [ba] y [bu], por ejemplo, comparten el elemento inicial mientras que [ba] y [ta] tienen el elemento final común. Los lingüistas llaman fonos o fonemas ${ }^{4}$ a estos segmentos de sílaba. El sistema alfabético utiliza signos que corresponden grosso-modo a los fonemas. Esto representa una economía considerable, puesto que de 20 a 30 signos bastan para representar toda la lengua. La reducción progresiva del número de signos cuando se pasa del chino logográfico al japonés silábico, y de éste al alfabeto nuestro va emparejada con un aumento de la complejidad de la relación que existe entre los signos ortográficos y los segmentos de la lengua correspondiente (Gleitman y Rozin, 1977). Para comprender el principio logográfico hay que ser capaz de identificar en el discurso oral los segmentos correspondientes a los logogramas, aproximadamente las palabras. Esta operación es mucho más fácil que la operación implicada en la identificación de los segmentos que corresponden a las letras del alfabeto, es decir, los fonemas. Este problema será examinado más adelante en detalle. El punto importante por el momento es que los sistemas fonográficos, tanto silábicos como alfabéticos, permiten el acceso al léxico por medio de la vía fonológica o indirecta. Un individuo que conoce el ocódigo puede crear una representación fonológica dé la palabra escrita utilizando, según el caso, reglas de transformación grafema-sílaba o grafema-fonema y con la representación así obtenida acceder al léxico interno, es decir, al significado. La existencia de esta posibilidad no es, por lo menos a priori, incompatible con la utilización de la vía directa. Se puede concebir perfectamente un sistema en el cual las dos vías funcionen paralelamente. El significado es alcanzado indistintamente por una $u$ otra según una serie de factores como el carácter más o menos familiar de la palabra y el estado del léxico interno. Por ejemplo, el contexto puede facilitar la evocación de ciertas entidades lexicales gracias al fenómeno llamado de "priming» (ver, por ejemplo, Meyer y Schvaneveldt, 1971). La vía directa en un sistema alfabético sólo puede ser desarrollada gracias a la repetición. Una palabra encontrada por primera vez no puede ser tratada como un logograma, puesto que no existe ninguna representación en el léxico interno que corresponda y pueda así ser evocada directamente por la palabra escrita. El lector está obligado, en este caso, a crear una representación fonológica y con ella acceder al léxico. Este proceso va a contribuir a crear una representación ortográfica en el léxiço interno que permitirá ulteriormente el acceso directo. Así, cada vez que el lector encuentra la misma palabra, la probabilidad de que la via directa permita identificarla se ve aumentada. Es importante recordar aqui que el letrado chino, al cual hicimos previamente alusión, no tiene más recurso que el de abrir el diccionario para identificar un logograma desconocido u olvidado, puesto que sólo dispone de la vía directa. La utilización simultánea y combinada de las vías directa e indirecta permite el auto aprendizaje en 
la medida en que la vía indirecta hace del niño (o del adulto) un lector autónomo, es decir, alguien que puede identificar una palabra que conoce fonéticamente, pero que no ha visto nunca antes por escrito.

\section{VIAS DE ACCESO AL LEXICO, METODOS DE APRENDIZAJE Y DIFICULTADES DE LECTURA}

Los métodos de aprendizaje de la lectura pertenecen a dos grandes familias. Los llamados fónicos o sintéticos, que comienzan por presentar al niño la correspondencia letra-fono o fonema y los globales o analíticos que hacen como si (por lo menos al principio) las palabras escritas fueran logogramas. Se supone más o menos explícitamente que los primeros favorecen el desarrollo de la vía indirecta y los segundos el de la vía directa. Muchas discusiones entre partidarios de uno u otro método, sin embargo, no toman en cuenta el hecho de que el niño se transforma en un lector hábil leyendo. El hecho de que su profesor le ayude a comprender la relación que existe entre las letras y los segmentos de palabra que representan no va a impedirle crear la vía directa al significado, sino al contrario, va a permitirle fabricársela él solo si tiene la motivación para leer. Por otro lado, la insistencia en los. aspectos puramente abstractos, es decir, lejanos al significado, del código alfabético, puede tener efectos negativos a nivel de la motivación del niño, del mismo modo que el solfeo aleja a muchos del aprendizaje de la música. Un razonamiento basado en consideraciones de este tipo puede hacer preferir un método a otro. El problema de la motivación, que es un problema central a nivel escolar, está fuera de mi propósito (y de mi competencia) al escribir este artículo destinado a discutir los problemas perceptivos y psicolingüísticos relacionados con la adquisición de la lectura.

La idea de que aprender a leer consiste en remplazar el modo laborioso y lento del comienzo, asociado a la utilización de la vía indirecta, por otro rápido que resulta de la utilización de la vía directa, es, en el mejor de los casos, incompleta. Un gran número de trabajos recientes muestran que el lector hábil no abandona la vía indirecta. Más importante aún, la diferencia principal entre buenos y malos lectores se sitúa a nivel de la utilización de esta vía, los buenos lectores la utilizan más eficazmente que los malos, que se ven así forzados a utilizar la vía directa.

Un argumento importante que pone en evidencia la utilización de la via fonológica en el lector adulto (estudiantes universitarios) viene de los trabajos de Rubenstein, Lewis y Rubenstein (1971). El sujeto mira una pantalla donde aparece una secuencia de letras durante un tiempo breve. Las letras pueden formar una palabra, brain, o una pseudopalabra, slint. La tarea del sujeto es decir rápidamente si se trata de una palabra o no. El resultado que nos interesa aquí es que la respuesta "no" a pseudopalabras homófonas de palabras, brane, por ejemplo, es más lenta que la respuesta «no» a pseudopalabras no homófonas, slint. Este resultado muestra que la decisión sí/no es tomada teniendo en cuenta la versión fonológica de la secuencia de letras a juzgar. La idea de que los malos lectores leen mal porque recurren más a la vía indirecta que los buenos lectores permite prever que en una experiencia de decisión lexical como la descrita, el efecto de 
homofonia será más importante en los malos que en los buenos lectores. Los resultados muestran exactamente lo contrario. Los buenos lectores presentan un efecto de homofonía sustancial, mientras que en los malos lectores, el efecto es nulo (Perfetti y Hogaboam, 1975) (ver también Perfetti, Goldman y Hogaboam, 1979) en una serie de experiencias destinadas a comparar buenos y malos lectores de tercer y quinto año seleccionados con un test de comprensión, han demostrado claramente que los buenos lectores se diferencian de los malos sobre todo en situaciones que hacen intervenir la vía indirecta y mucho menos en aquellas en que la vía directa juega el papel más importante. El factor experimental utilizado por estos autores es la frecuencia. El sujeto tenía que leer en voz alta palabras de alta frecuencia, de baja frecuencia o bien pseudopalabras. Es razonable suponer que la vía directa interviene de manera masiva en la identificación de palabras frecuentes. La pronunciación se obtiene, en este caso, a nivel pos-lexical, del mismo modo que el código fonológico [dos], por ejemplo, es evocado después de haber identificado de manera directa la cifra 2, o que el código fonológico [pato] es evocado después de haber visto un pato, la foto de un pato o haber pensado en Donald. Las pseudopalabras no pueden ser identificadas directamente. Para leerlas hay que pasar obligatoriamente por la aplicación de reglas de transformación grafema-fonema. En cuanto a las palabras de baja frecuencia, su estatuto es intermediario entre el de las palabras de alta frecuencia y el de las pseudopalabras. La idea de que los malos lectores sólo emplean la vía indirecta, es decir, que pasan necesariamente por la traducción grafema-fonema conduce a la hipótesis de que la diferencia con los buenos lectores será pequeña o nula leyendo pseudopalabras y más importante leyendo palabras de alta frecuencia.

Los resultados son diametralmente opuestos. Los buenos lectores son más rápidos leyendo en voz alta que los malos lectores, pero la diferencia es relativamente pequeña con palabras de alta frecuencia $\mathbf{2 2 2}$ y $174 \mathrm{~ms}$ en tercer y quinto año, respectivamente), aumenta considerablemente al pasar a palabras de baja frecuencia ( 1.073 y $1.015 \mathrm{~ms}$ ) y alcanza el máximo con pseudopalabras (1.159 y $1.222 \mathrm{~ms}$ ). Este resultado muestra también que los buenos lectores se caracterizan por una utilización más eficaz de la vía indirecta que los malos lectores y mucho menos a nivel de la vía directa.

En resumen, los resultados expuestos en los últimos parágrafos sugieren que las dos vías posibles de acceso al léxico que se pueden concebir en un sistema alfabético funcionan paralelamente y que la idea de que la vía directa reemplaza la indirecta en los buenos lectores es incorrecta. La comparación entre buenos y malos lectores indica, al contrario, que la ventaja de los primeros no resulta de una utilización más frecuente o más eficaz de la vía directa, sino más bien de la indirecta: El origen de esta diferencia podria situarse a nivel de la comprensiór del principio alfabético.

\section{s. ADQUISICION DEL CODIGO ALFABETICO}

El código alfabético, a diferencia de otros sistemas de escritura, representa la palabra a nivel de fonos y fonemas. En la medida en que para leer bien hay que poder explotar las dos vías de acceso al léxico 
interno, un buen lector tiene necesariamente que haber identificado los segmentos de palabra que corresponden a las letras. Sin esta condición no podrá desarrollar el sistema de reglas que permiten crear un código fonológico a partir de la representación ortográfica. Este sistema constituye el elemento central de la vía indirecta. Una deficiencia a este nivel tendrá, probablemente, repercusiones en el desarrollo de la vía directa también, puesto que la indirecta es un elemento importante en el proceso de autoaprendizaje.

Para un adulto, familiarizado con el código alfabético, el hecho de que palabras como cal y sal, por ejemplo, estén constituidas de tres segmentos y que sólo el primero permita distinguir una de otra o bien que sapo, sota, silaba y sufre comiencen todas con el mismo segmento, son hechos evidentes. Para el niño de cinco o seis años que comienza el aprendizaje de la lectura, esta realidad es mucho menos evidente que para su profesor. Hasta entonces, el lenguaje para él transmitia información, y su estructura fonética no tenía ningún interés. El niño de cuatro años distingue perfectamente entre cal y sal en un plano semántico; si oye decir que hay que ponerle «cal a la sopa» mirará sin duda, extrañado, al autor de la frase o pensará que oyó mal. Pero esta capacidad resulta de la intervención de sistemas automáticos de tratamiento fonético en los cuales la conciencia no interviene. Tomar conciencia del hecho de que cal y sal están compuestos de tres segmentos fonéticos es completamente inútil para comprender la lengua oral. La idea que quisiera defender en este parágrafo es que esta toma de conciencia es indispensable para adquirir el código alfabético $\mathrm{y}$, por consiguiente, para leer bien.

¿Cómo llega el niño a tomar conciencia de la estructura fonética de la palabra? Para responder a esta pregunta hay que examinar su capacidad de aislar conscientemente segmentos de palabra. El grupo de Haskins (Liberman, Shankweiler, Fischer y Carter, 1974) ha demostrado que niños de cuatro y cinco años son capaces de segmentar palabras en sílabas, pero no en fonos. Los sujetos debían, después de haber oído una palabra pronunciada por el experimentador, dar en la mesa tantos golpes como sílabas (o fonos) tenía la palabra. Para realizar esta tarea el niño tiene que aislar mentalmente los segmentos, sílabas o fonos, contarlos y dar su respuesta. A los seis años, es decir, al empezar el aprendizaje de la lectura, la capacidad de segmentación en fonos aumenta bruscamente. Estos resultados confirman otros menos sistemáticos (Rosner y Simon, 1971; Leroy-Boussion y Martínez, 1972; Calfee, Chapman y Venezky, 1972; Zhurova, 1973) y han sido ampliamente confirmados ulteriormente. La diferencia de dificultad que se observa entre fonos y sílabas proviene probablemente del hecho de que a las silabas corresponde un parámetro físico simple. El análisis acústico del mensaje oral muestra, en efecto, una sucesión de picos de intensidad que corresponde casi perfectamente a los núcleos vocálicos de las sílabas. El sujeto, para aislar una sílaba, puede utilizar este parámetro con relativa facilidad. Esta es la razón por la cual los niños de cuatro y cinco años son capaces de realizar tareas que implican la manipulación de sílabas, que son el más aparente de los segmentos de palabra que carecen de significado. Por esta misma razón, los sistemas de escritura que utilizan la silaba como unidad, el Kana japonés en particular, son más accesibles que los sistemas alfabéticos. Según Makita (1968), Sakamoto y Makita (1973) y Sakamoto (1980), los niños japoneses 
aprenden el Katakana antes de entrar a la escuela, sin enseñanza organizada sistemáticamente. Estos autores agregan que las dificultades escolares debidas a la lectura son raras en Japón. El argumento es importante, pero hay que tomarlo con precaución, porque la noción de dificultad o de fracaso escolar es una noción administrativa y no científica. (Véase, por ejemplo, Stevenson, Stigler, Lucker y Lee, 1982). $\mathrm{El}$ análisis de sílabas en segmentos fonéticos es más complejo que el de palabras en sílabas porque no existe ningún fenómeno acústico específico que corresponda a cada segmento fonético. Los fonos son transmitidos paralelamente, es decir, que a cada instante la corriente acústica transmite información sobre varios fonos simultáneamente (A. Liberman, 1970). Por esta razón, la toma de conciencia de la estructura fonética de la palabra $y$, por consiguiente, la comprensión del código alfabético, plantea problemas a ciertos niños. Estos problemas son especificos de la lectura y no intervienen en la producción y en la comprensión del lenguaje.

En un trabajo reciente (Morais, Cluytens y Alegria, 1984) un grupo de 27 niños disléxicos fue sometido a tareas de manipulación explícita de fonos y de sílabas. Su edad variaba entre los seis años y un mes, y los nueve años y seis meses (media ocho años). Estos niños asistían a una escuela especialmente destinada a niños con problemas de lectura. Todos tenian una puntuación normal en el WISC (éste es uno de los criterios de admisión de la escuela). Un test de lectura que consistia en leer en voz alta una lista de palabras regulares, mono y bisilábicas, da como resultado 3,4 palabras leidas en un minuto. Los resultados de dos grupos control, uno de primero y otro de segundo año primario, de clases ordinarias, son 19,3 y 28,8 palabras, respectivamente. La tarea de segmentación de los sujetos consistía en extraer el fono inicial de una expresión pronunciada por el experimentador. El fono podía tener valor silábico [a] (el experimentador decía atu y el niño tenía que responder $t u$, o bien valor fonético: [p] o [f] (pal $\rightarrow a l, f u r \rightarrow u r$, etc.). En este último caso, los disléxicos alcanzan 13,7 por roo de respuestas correctas. Los grupos control de primer y segundo año obtienen $7 \mathbf{1} .3$ y 94.6 por 100 , respectivamente. La diferencia es considerable. Los resultados a nivel silábico son menos dramáticos: 68,3 por 100 en los disléxicos y superiores a 90 por 100 en los niños de escuela normales. Los sujetos fueron sometidos también a un test con material no lingüístico que consistía en sustraer la primera de cuatro notas ejecutadas por el experimentador en un xilófono de juguete. Sólo se utilizaban los «dos» extremos para simplificar la discriminación. Las otras notas estaban cubiertas por un cartón. Los resultados dan 28,8 por 100 de respuestas correctas en los disléxicos contra 16,6 por 100 y 39 , I por 100 en los niños de primer y segundo año, respectivamente. La habilidad de los disléxicos para segmentar notas musicales es prácticamente normal. Sus dificultades se sitúan especificamente a nivel de la segmentación de la palabra en fonos y no de la segmentación de secuencias de sonidos en general.

Un argumento suplementario que indica que los disléxicos tienen dificultades con el código alfabético proviene de los trabajos de Rozin, Poritsky y Sotsky (1971). Estos autores tomaron un grupo de niños de segundo año de una escuela del "ghetto» negro de Filadelfia, con dificultades graves en lectura. Ninguno de ellos era capaz de leer trigramas como: jip, zif, wat, etc. Eran incapaces también de leer series 
de palabras muy corrientes en inglés que riman en [at]: cat, fat, rat, hat, etc. Los niños fueron sometidos a dos o tres sesiones semanales de entrenamiento (en total ocho a catorce horas). La tercera parte del tiempo era consagrado al aprendizaje normal de la lectura y los dos tercios restantes a aprender 26 caracteres chinos. Al final de este periodo los progresos en lectura en inglés eran nulos, pero los niños eran capaces de leer frases escritas con los caracteres chinos que habian aprendido. Este resultado es importante por varias razones. En primer lugar, demuestra que los niños de esta experiencia no tenían problemas para asociar 26 signos gráficos a los segmentos de lenguaje correspondientes cuando los signos representan palabras. La adquisición del principio logográfico se hace en pocas horas. La dificultad concierne la adquisición del principio alfabético, porque, probablemente, estos niños, como los disléxicos examinados por Morais et al. (1984), no son capaces de aislar los segmentos fonéticos de palabra a los que corresponden las letras.

La adquisición del código alfabético no es un proceso brutal de tipo todo o nada. Ciertos segmentos fonéticos son más fáciles de aislar que otros y la dificultad relativa depende igualmente del lugar que ocupan en la expresión (Content, 1984; Content, Kolinsky y Morais, 1984). Las vocales, por ejemplo, son más fáciles de aislar que las consonantes, porque pueden funcionar como sílabas y/o porque pueden ser pronunciadas solas. Las oclusivas [b, d, g, p, t, k] son, probablemente, las más difíciles de aislar, porque son las más sensibles al contexto vocálico en el cual aparecen. El análisis espectrográfico de las sílabas [di, de, d $\mathbf{E}$, $\mathrm{da}, \mathrm{d} \supset, \mathrm{do}, \mathrm{du}]$ muestra que no existe ningún invariante acústico inicial que corresponda al fondo [d]. Las dificultades que tienen muchos niños con las letras b, p; d, q, g, no provienen fundamentalmente de problemas visuo-espaciales (ver parágrafo 2 ). La dificultad principal es que los segmentos de palabra correspondientes son los más dificiles de aislar. Si a esto se agrega el hecho de que son semejantes unos de otros desde el punto de vista acústico: [b] es acústicamente más próximo de [p] y de [q] que de [s] y de [l]; por ejemplo, y, finalmente, que las representaciones ortográficas son también similares se puede comprender porque los niños de seis, siete y a veces más años tienen problemas con ellos sin que esto tenga ningún carácter patológico. La situación se parece a la de un profesor que tiene que aprender los nombres de sus alumnos, es decir, asociar niño-nombre, y que se encuentra con cuatro o cinco que se parecen físicamente y que tienen nombres parecidos también. Evidentemente la tarea sería más fácil si Roberto fuera gordo y Norberto flaco y/o si siendo físicamente parecidos, uno de los dos se llama Juan. El problema del niño con las oclusivas es más serio aún que el del profesor con sus alumnos, porque a la similitud visual entre las letras y acústica entre los fonos se agrega la dificultad de aislar los fonos del contexto silábico donde aparecen. El profesor no tiene problemas para «aislar» perceptivamente los niños del contexto «clase» donde se encuentran.

Estos resultados muestran que el acceso al código fonético plantea problemas a muchos niños y que esto va a la par con dificultades serias para aprender a leer en un sistema alfabético. Para poder ayudar a estos niños sería útil comprender cómo los otros, los que toman conciencia de la estructura fonética de la palabra y que, afortunadamente, repre- 
donde yo estoy integrado trabaja sobre este problema desde hace varios años. Desgraciadamente, tenemos que reconocer que no podemos dar una respuesta categórica a esta pregunta. Un hecho, al menos, ha podido ser claramente establecido. El ser humano no llega espontáneamente a descubrir la estructura fonética de la lengua. Para que esto ocurra, una intervención externa es indispensable y esta intervención es generalmente el hecho de tener que aprender a leer en un sistema alfabético (Alegria y Morais, 1979). Esto ha sido demostrado de tres formas diferentes. En primer lugar comparando la habilidad a manipular fonos en niños de la misma edad, seis años, aprendiendo a leer con el método fónico o con el método global. Sólo los primeros, es decir, aquellos en presencia del código alfabético, son capaces de hacer operaciones con fonos (Alegría, Pignot y Morais, 1982). La segunda demostración proviene de resultados de segmentación obtenidos con adultos analfabetos (Morais, Cary, Alegria y Bertelson, 1979). Si la capacidad de manipular segmentos fonéticos se desarrolla espontáneamente; los analfabetos serian capaces de hacer operaciones de sustracción y de adición de fonos. Los resultados muestran que son incapaces. Finalmente Read, Zhang, Nie y Ding (1984) examinaron dos grupos de adultos chinos que habían hecho aproximadamente diez años de escuela. Un grupo había aprendido el pin-yin ${ }^{5}$ y otro no. La tarea que Read et al. propusieron a sus sujetos es la misma que la de Morais et al., 1979, respecto a los analfabetos. Los resultados muestran que sólo los sujetos que habian sido confrontados con el código alfabético (pin-yin) eran capaces de hacer operaciones de sustracción y de adición de fonos.

Estos resultados conducen a una conclusión en apariencia circular: para aprender a leer en un sistema alfabético hay que ser capaz de manipular los segmentos fonéticos de la lengua y para manipular los segmentos fonéticos de la lengua hay que aprender a leer en un sistema alfabético. La circularidad es sólo aparente. Aprender a leer, como propuse anteriormente, es una tarea compleja que consiste en integrar la "lengua visual» en el proceso de tratamiento de la lengua oral que preexiste y funciona perfectamente. El resultado final, leer bien, puede no ser alcanzado por diversas razones. Leer bien es una habilidad total que engloba una serie de habilidades componentes. Una de ellas, importante, pero no la única, es la habilidad de análisis fonético de la palabra. La relación entre la lectura y el análisis fonético es una relación de tipo todo-parte. Para adquirir la habilidad total, cada uno de los componentes tiene que ser adquirido y, por consiguiente, los problemas a nivel de uno y otro de los componentes va a crear dificultades en la adquisición del todo (Alegria y Content, 1983). Una serie de trabajos recientes llevados a cabo por Alain Content demuestra que es posible, y relativamente fácil en la mayoría de los casos, desarrollar la habilidad de análisis fonético en los niños de cinco años y algo menos en los de cuatro, empleando tareas que los divierten, y esto de manera totalmente independiente de la lectura (Content, Morais, Alegria y Bertelson, 1982; Content, Kolinsky, Morais y Bertelson, 1984). Estos trabajos podrian tener un cierto impacto pedagógico en la medida en que separan al problema del aprendizaje de la lectura del de la comprensión del código alfabético. Queda por ver, y esto es probablemente lo más delicado, si esto ayuda a los niños que, por razones que no entendemos, no consiguen acceder al código alfabético. 
En resumen, el examen de los procesos implicados en la lectura conduce a la idea de que las dificultades que se plantean para aprender a leer no son de naturaleza perceptiva. El niño que aprende a leer con dificultad, que confunde ciertas letras (b y p, por ejemplo) no lo hace, en la gran mayoría de los casos, porque tiene una organización espacial deficiente y confunde la derecha y la izquierda. El problema principal es de orden lingüistico. Para aprender a leer hay que analizar la lengua a un grado de profundidad que depende del código ortográfico al cual se está confrontando. En el caso del alfabeto, el niño tiene que tomar consciencia de la estructura fonética de la lengua.

Un número importante de dificultades de lectura se sitúan a este nivel y si bien no estamos en condiciones de dar recetas sobre la manera en que se puede ayudar al niño que no llega sólo, está claro que los esfuerzos tienen que ser dirigidos en la dirección del análisis de la lengua. En este artículo nos hemos limitado a discutir el análisis a nivel fonético, pero es evidente que los otros niveles intervienen también, aunque en castellano el problema sea relativamente menor. En francés, por ejemplo, el análisis fonético es insuficiente porque la ortografia es menos fonética que en castellano y más morfológica. Para leer (y sobre todo para escribir) el niño tiene que hacer un análisis gramatical. Por ejemplo, todos los homófonos del infinito de los verbos en -er (los más frecuentes) se escriben de manera diferente pero se pronuncian igual. El niño, para saber si $\left[\operatorname{mar} S_{\varepsilon} / e\right]^{6}$ se escribe marcher, marché, marchais, marcbait, marcbaient, marchez o marchai tiene inevitablemente que analizar la frase. Es decir, descubrir si el verbo está conjugado o bien si está en infinitivo: marcher. Si está conjugado tiene que preguntarse si tiene o no auxiliar, en cuyo caso se trata del participio: marché. Si no tiene auxiliar hay que determinar el sujeto: si se trata de je o $t u$, marcbais; si es $i l$, marcbait, y si es ils: marchaient. Il y ils son también homófonos y por consiguiente el análisis de la frase tiene que llegar más lejos para sāber si el sujeto es singular o plural. Para decidir si se trata de marchai, pasado simple, o marchais, imperfecto, hay que entrar en consideraciones semánticas para saber si la acción tiene lugar en un momento preciso del pasado o no. Este ejemplo muestra que el análisis fonético no es suficiente para dominar la lecto-escritura. Lo tomo del francés porque es una lengua cuya morfología es mucho más transparente a nivel ortográfico que a nivel oral. En castellano, el problema para el lector debutante es menos serio porque la morfología es relativamente transparente ya a nivel oral. La lectura agrega, por tanto, menos que en francés. Es razonable suponer que una proporción importante de los problemas de adquisición de la lectura en castellano, dependa directamente de la comprensión del código alfabético. 
1 El autor de este artículo trabaja en el seno de un equipo dirigido por el profesor Paul Bertelson, que se interesa, entre otras cosas, por la psicologia cognitiva de la lectura. Aunque todo lo dicho sea de la responsabilidad exclusiva del autor nada hubiera sido posible sin el sostén intelectual de cada uno de los miembros del equipo. Uno de ellos, José Morais, merece un reconocimiento especial por haber aceptado leer y comentar una versión previa del artículo.

2 Un examen crítico de los trabajos y especulaciones de Orton, Hermann, Satz, etc., en este dominio puede ser leido en Vellutino (1979), capitulo 3.

3 Conjuntamente con los dos silabarios, el Hiragana y el Katakana, los japoneses utilizan un sistema logográfico importado de China, llamado el Kanji.

4 Los fonos son los sonidos elementales de la lengua a nivel oral. Dos fonos son considerados diferentes uno del otro si la diferencia es perceptible. En mi dialecto del castellano, por ejemplo, el sonido representado por $s$ en sopera y en espera son diferentes. Los morfemas están representados en el léxico interno del sujeto como secuencias de fonemas que tienen un carácter abstracto y no un carácter material (acústico) como los fonos. El mismo fonema puede dar lugar a fonos diferentes según el contexto, como en el ejemplo de aquí arriba. Una discusión excelente de las relaciones entre fonemas y fonos puede ser consultada en Gleitman y Rozin (1977).

5 Método alfabético introducido por Mao en 1949 destinado a facilitar la redacción de textos destinados a los extranjeros: cartas geográficas, nombres propios, etc. En general, los niños aprenden el pinyin en la escuela primaria y lo olvidan por falta de uso.

$6 \mathrm{La}$ distinción entre $\left[\operatorname{mar} \mathcal{S e}_{\mathrm{e}}\right.$ y $\left[\operatorname{mar} \mathcal{S}_{\varepsilon}\right]$ tiende a desaparecer en "francés central», pero sigue practicándose en «francés periférico», en Bélgica por ejemplo.

\section{Referencias}

ALEGRIA, J.: Estructura del lenguaje, alfabeto y aprendizaje de la lectura. Barcelona, en prensa.

Alegria, J., y Morais, J.: "Le développement de l'habilité d'analyse phonétique de la parole et l'apprentissage de la lecture». Archives de Psychologie, 1979, 47, 25 1-270.

Alegria, J., PIGnot, E., y Morais, J.: «Phonetic analysis of speech and memory codes in beginning readers». Memory and Cognition, 1982, 10, 45 1-456.

AlegriA, J. y CONTENT, A.: Explicit analysis of speecb and learning to read: some experimental studies. Comunicación presentada a la 3 rd. Language y Language Acquisition Conference on «Pragmatics and Education». Gent, $21-2$; marzo 1983.

BARON, J. y TREIMAN, R.: "Use of orthography in reading and learning to read»: En J. F. Kavanagh y R. L. VENEZKY : (Eds.) Ortbography, Reading, and Dyslexia. Baltimore, Univ. Park Press, I980.

Calfee, R. C.; Chapman, R. S., y Venezky, R. L.: «How a child needs to think to learn to read». En L. W. Gregg (Ed.) Cognition in learning and memory. Nueva York, Wiley, 1972.

Coltheart. M.: "Lexical access in simple reading tasks». En G. Underwood (Ed.) Strategies of information processing. Londres, Academic, Press, 1978.

CONTENT, A.: «Le développment de l'habilité d'analyse phonétique de la parole». L'Année Psycbologique, en prensa, 1984 .

Content, A.: "L'habilité d'analyse phonétique et l'acquisition de la lecture». L'Année Psycbologique, en prensa, 1984 .

Content, A.; Morais, J.; Alegria, J., y Bertelson, P.: «Accelerating the development of phonetic segmentation skills in kindergartners». Cabiers de Psycbologie Cognitive, 1982, 2, 259 -269.

CONTENT, A.; Kolinsky, R.; MORAIS, J., y BERTElson, P.: «Access to phonetic awareness in prereaders». Manuscrito en preparación, 1984.

ELLIS, N.: "Visual and name coding in dyslexic children.» Psycbological Research, 1981, 43, $201-218$.

GLEITMAN, L. R., y RoziN, D.: «The structure and acquisition of reading, 1 . Relation between orthographies and the structure of language». En A. S. Reber y D. L. Scarborough (Eds.) Towards a prycbology of reading. Nueva York, John Wiley y Sons, 1977.

JORM, A. F. y SHARE, D. L.: «Phonological recoding and reading acquisition». Applied Psycbolinguistics, $1984,4,103-147$.

LEROY-BOUSSION, A., y MARTínez, F.: UUn prérequis auditivo-phonétique pour l'apprentissage du language écrit: l'analyse syllabique. Etude génétique longitudinale entre, et 8 ans»). Enfance, 1972, 8, $111-130$.

Liberman, I. Y.: "A language-oriented view of reading and its disabilities». En $H$. Myklebust (Ed.) Progress in learning disabilities. Vol. s. Nueva York, Grune y Stratton, 1981 .

Liberman, A. M.; CoOper, F. S.; Shankweiler, D. P., y Studdert-Kennedy, M.: «Perception of the speech codes. Psycbological Review, 1967, 74, 431-461.

Liberman, I. Y.; Shankweiler, D.; Fischer, F. W., y Carter, B.: «Reading and the awareness of linguistic segments". Josrnal of Experimental Cbild Psycbology, 1974, 18, $201-212$.

MAkiт A, K.: «Rarity of reading disability in japanese children». American Journal of Orthopsichiatry, 1968, 38, $599-614$.

MeYER, D. E. y SChVANEveldT, R. W.: «Facilitation in recognizing pairs of words: Evidence of dependence between retrieval operations». Journal of Experimental Psychology, 1971, 90, 227-234.

Morais, J.; Cary, L.; Alegria, J., y Bertelson, P.:«Does awareness of speech as a sequence of phones arise spontaneously?" Cognition, 1979,7,323-331.

Morais, J.; Cluytens, M., y Alegría, J.: "Segmentation abilities of dyslexics and normal readers." Perceptual and Motor Skills, 1984, $58,221-222$. 
Perfetti, C. A. y Goldman, S. R.: «Discourse memory and reading comprehension skill». Journal of Verbal Learning and Verbal Bebavior, 1976, 14, 33-42.

PerfetT, C. A.; Goldman. S., y Hogaboom, T.: "Reading skill and the identification of words in discourse contexts. Memory and Cognition, 1979, 7, 273-282.

POSNER, M. I.: "Abstraction and the process of recognition". En J. T. Spencer y G. Bower (Eds.) Tbe psycbology of learning and motivation, Vol. 3. Londres, Academic Press, I 969.

ReAD, C.; Zhang, Y.; NIE, H., y Ding, B.: "The ability to manipulate speech sounds depends on knowing alphabetic spelling». Manuscrito, 1984.

POSNER, J. y SimON, D. P.: Tbe auditory analysis test: An initial report learning researcb and development center, University of Pittsburgh. Publication, 1971/3.

Rozin, P.; PORITSKY, S., y SOTSKY, R.: "American children with reading problems can easily leam to read english represented by chinese characters». Science, 1971, $71,1.264-1.267$.

RUBENSTEIN, H.; LEwis, S. S., y RUBENSTEIN, M. A.: «Evidence for phonemic recoding in visual word recognition». Journal of Verbal learning and Verbal Bebavior, 1971, 10, 645-657.

SAKАмOTо, T.: «Reading of Hiraganam. En J: F. Kavanagh y R. L. Venezky (Eds.) Ortbograpby, Reading, and Dislexia, Baltimore, University Park Press, 1980.

SAKAMOTO, T. y MAKITA, K.: Japan. En J. Dowing (Ed.) Comparative reading: Cross-national studies of bebavior and processes in reading and עriting. Nueva York, Macmillan, 1973.

Vellutino, F. R.; Pruzek, R.; Steger, J. A., y Meshoulam, V.: uImmediate visual recall in poor and normal readers as a function of orthographic-linguistic familiaritym. Cortex, 1973, 9, 368-384.

Vellutino, F. R.: Dyslexia: Theory and researcb. Cambridge, Mass., M.I.T. Press, 1979.

Vellutino. F. R.; STEGER, J. A., y KANDEI, G.: «Reading disability: An investigation of the perceptual deficit hypothesis.y Cortex, 1972, 8, 106-118.

Vellutino, F. R.; Steger, J. A.; Kaman, M., y De Setto, L.: “Visual form perception in deficient and normal readers as a function of age and orthographic-linguistic familiarity". Cortex, 1975, 11, 22-30.

ZHUROVA, L. Y.: "The development of analysis of words into their sounds by preschool children». En C. A. Ferguson y D. I. Slobin (Eds.) Studies of cbild language development. Nueva York, Holt, Rinehart $y$ Winston, 1973.

\section{Resumen}

El anailisis del acto lectura nos lleva a proponer que aprender a leer consiste en descubrir cómo utilizar el dispositivo destinado inicialmente a comprender frases orales para comprender las mismas frases en presentación escrita. Los resultados de experiencias con niños que tienen dificultades para aprender a leer muestran que los problemas no son de naturaleza perceptivo-visual ni de organización del espacio, sino de nominación de configuraciones visuales. Examinamos enseguida las diferentes vias de acceso a los conocimientos lexicales del individuo, $y$ en particular las dos vias posibles a partir de la representación escrita de la palabra: la vía directa y la via indirecta o fonológica, y sus relaciones con el aprendizaje de la lectera. La idea de que los malos lectores son malos porque utilizan preferencialmente la via fonológica puede ser eliminada. Los trabajos sugieren que, contrariamente a una opinión muy corriente, son los malos lectores los que tienen problemas para utilizar la via indirecta. Finalmente, se discuten las relaciones posibles entre el análisis explicito de la palabra, la comprensión del código alfabético y el desarrollo de las vias de acceso al léxico interno a partir del texto.

\section{Summary}

The analysis of the reading act brings us to propose that learning to read consists into find a way to use the device initially developped to understand oral sentences, to understand the same sentences in an ortbograpbic representation. Experimental data obtained with children presenting reading acquisition troubles shows that their problems are neitber visuo-perceptual nor spatial ones, but that they are related to naming visual patterns. We examine then the different ways allowing the subject to access to the lexical information be bas stored in memory, and particularly, the two possible patbs from the written representation of speech: the direct and the indirect or phonological one, and their relationships with reading acquisition. The idea that poor readers are poor because tbey prefer the pbonological patbway can be eliminated. The evidence suggest indeed that poor readers cannot adeguatly use tbe phonological patbway. Finally we discuss the possible relations between the explicit analysis of speech, the understanding of the alpbabetic principle asd the development of the pathways to the lexicon from the orthographic representation of speech.

\section{Résumé}

L'analyse l'acte de lecture nous mine à proposer que apprendre à lire consiste à découvrir la façon d' utiliser le dispasitif initialement destiné à comprendre des phrases orales, pour comprendre les mémes pbrases présentées par écrit. Les résultats des experiences portant sur des enfants qui ont des difficultés pour apprendre à lire montrent que leurs problemes ne sont pas de nature perceptivo-visuelle ni d'organisation de lespace mais plutôt de nomination de configurations visuelles. Nous examinons ensuite les différentes voies d'actès aux connaissances lexicales du sujet et en particulier des deux voies possibles a partir de la representation écrite de la parole: la voie directe et la voie indirecte ou pbonologique, et leurs rapports avec a papprentissage de la lecture. L'idée que les mauvais lecteurs seraient mauvais parce qu'ils ufilisent préférentiellement la voie phonologique est écartée. Les travaux suggèrent que, contrairement à une idée très répandue, ce sont les mavvais lecteurs qui ont des problèmes avec la voie phonologique. Finalement nous discutons des rapports possibles entre l'analyse explicite de la parole, la comprébension du code alpbabétique et le développement des voies d'accés au lexique as lexigue à partir du texte. 